\title{
Nuevas metodologías docentes aplicadas al estudio de la fisiología y la anatomía: estudio comparativo con el método tradicional
}

\author{
B. Gal-Iglesias a , I. de Busturia-Berrade b, M.C. Garrido-Astray ${ }^{a}$
}

Introducción. El estudio integrado de la fisiología y la anatomía constituye una asignatura troncal dentro de las titulaciones de ciencias de la salud. La adaptación de la enseñanza de esta materia al estándar de Bolonia supone un reto metodológico dada su complejidad curricular y sus perfiles competenciales específicos. Objetivos. Evaluar experimentalmente una metodología mixta en la enseñanza de la fisiología y anatomía tanto en los resultados del aprendizaje como en la adquisición de competencias de los alumnos, así como desarrollar un baremo de medición del aprendizaje. Sujetos y métodos. Se comparan los resultados del aprendizaje en la diplomatura de podología de la Universidad Europea de Madrid entre un grupo control (metodología clásica: lección magistral) y uno experimental (metodología mixta: problemas, casos clínicos, trabajo en grupo y lección magistral). Resultados. Se muestra que con una aproximación mixta los alumnos adquieren habilidades y competencias a lo largo del curso, estabilizándose el aprendizaje lo suficiente y equilibrándose las diferencias individuales, en contraste con el grupo control. Esta homogeneización del aprendizaje se manifiesta como una menor variabilidad de la nota media de los exámenes en el grupo experimental, e indica el soporte que supone el método mixto para el aprendizaje de los alumnos con mayores dificultades. Se detecta una fuerte regresión lineal entre la asistencia a clase y los resultados del aprendizaje, y se confirma la importancia de la supervisión del proceso de aprendizaje por parte del profesor. Conclusión. El método mixto de enseñanza de la fisiología y la anatomía permite a los alumnos adquirir habilidades y competencias a lo largo del curso, equilibrando las diferencias como parte del proceso de aprendizaje.

Palabras clave. Anatomía. Aprendizaje basado en problemas. Competencias. Docencia. Fisiología. Innovación.

\section{Novel methodologies applied to Physiology and Anatomy teaching: comparison with traditional teaching}

Introduction. The integrated study of Anatomy and Physiology is mandatory in Health Sciences careers. The adaptation of these topics to the Bolonia paradigm in Europe is a methodological challenge due to the curricular complexity and specific competencies profiles involved. Aims. To evaluate a mixed methodology in the Physiology and Anatomy teaching aimed to measure knowledge and competencies. We also propose an evaluation scale to score learning. Subjects and methods. Data were gathered from a control group (classical methodology: lectures) which was compared with an experimental group (mixed methodology: problem-based learning $(P B L)$, clinical cases, team working and lectures) in the Podiatric School at the Universidad Europea de Madrid. Knowledge was measured with multiple choice exams and PBL, clinical cases and team working evaluation. Results. We show that the students gain knowledge and competences during the academic course using a new mixed methodology for teaching. In contrast to the control group, knowledge in the experimental group stabilizes over the academic course making individual differences between the students insignificant. This homogenization becomes evident as a lower variability of the average scores of the experimental group and gives support to the value of a mixed methodology for students with more difficulties. There is a strong linear regression between class attendance and exam scores, confirming the importance of face-to-face supervision for learning. Conclusions. A mixed methodology for Physiology and Anatomy teaching allows the students to acquire abilities and competencies, also helping to equilibrate individual differences during the learning process.

Key words. Anatomy. Competences. Innovation. Physiology. Problembased learning. Teaching.
Departamento de Ciencias Morfológicas, Fisiología y Biomedicina.

b Departamento de Especialidades Médicas, Psicología y Pedagogía Aplicadas. Universidad Europea de Madrid. Villaviciosa de Odón, Madrid, España.

\section{Correspondencia Dra. Beatriz Gal. Departamento de Ciencias Morfológicas, Fisiología y Biomedicina. Universidad Europea de Madrid. Tajo, s/n. E-28670 Villaviciosa de Odón (Madrid). \\ E-mail beatriz.gal@uem.es}

Trabajo financiado por el proyecto OTRI 2005/UEM04 de la convocatoria intramural 2005 de Proyectos de Investigación de la Universidad Europea de Madrid. 


\section{Introducción}

El aprendizaje no es una mera cuestión de memorizar, sino que consiste en la habilidad de utilizar recursos para encontrar, evaluar y aplicar la información. Sin embargo, los currículos densos, como los que se han venido utilizando, dejan poco tiempo al estudiante para una mejor comprensión de los conceptos o para desarrollar competencias como el pensamiento crítico, la resolución de problemas y el trabajo en equipo. Deberíamos, por tanto, reducir la cantidad de información que los estudiantes deben memorizar y promover que éstos fueran más activos e independientes a la hora de estudiar y resolver problemas [1].

La necesidad de abordar esta problemática en la sociedad actual, con una demanda creciente de estudiantes mejor formados para desarrollar perfiles profesionales específicos, hace que en el año 1999 se acordara la creación del Espacio Europeo de Educación Superior (EEES) (Bolonia, 1999) [2]. Esta declaración implica un cambio importante en los planteamientos de los docentes universitarios. La creación del EEES supone consensuar ideas sobre el concepto de la educación, el aprendizaje y la manera de cuantificarlo con el nuevo sistema de créditos ECTS (Sistema Europeo de Transferencia de Créditos, European Credit Transfer and Accumulation System). Esta reforma educativa supone una reflexión sobre la consecución de objetivos, competencias y conocimientos y la evidente adaptación de las metodologías docentes actuales [3].

El estudio integrado de la fisiología y la anatomía constituye una asignatura troncal dentro de las titulaciones de ciencias de la salud, y se imparte con denominaciones diferentes (estructura y función del cuerpo humano; morfología, estructura y función del cuerpo humano, etc.). El estudio y comprensión de esta disciplina es complejo, puesto que implica integrar conocimientos sobre los sistemas fisiológicos y anatómicos diferentes, y precisa de una adecuada base formativa (histología, bioquímica y biología). Por otro lado, el perfil profesional de un titulado de ciencias de la salud comprende la adquisición de competencias como razonamiento, capacidad de relacionar e integrar conocimientos, así como trabajar en equipo y la comunicación con el paciente. Sin embargo, muchos de los programas actuales no contemplan estos aspectos.
El equilibrio entre la adquisición de los conocimientos y la adquisición de las capacidades y habilidades resulta complicado de establecer. Por un lado, se ha demostrado que la clase magistral es efectiva en la transmisión de la información y en liderar el estudio $[4,5]$. Por otro, numerosos trabajos han probado que la utilización de metodologías diferentes, como la enseñanza basada en problemas, prácticas, y otro tipo de metodología participativa desarrollada en grupos [6,7] potencian la integración de los conocimientos adquiridos durante la lección magistral. Por lo tanto, se trataría de diseñar programas en los que se integren las metodologías diferentes en la proporción adecuada para maximizar tanto la adquisición de conocimientos como el desarrollo de competencias.

El objetivo principal de este trabajo ha sido evaluar experimentalmente una metodología mixta en la enseñanza de la fisiología y anatomía tanto en los resultados del aprendizaje como en la adquisición de competencias de los alumnos. Asimismo, se pretende desarrollar un baremo de medición del aprendizaje que no sólo evalúe la adquisición de conocimientos, sino de aquellas competencias vinculadas con esta asignatura.

\section{Sujetos y métodos}

Para evaluar el impacto de una aproximación metodológica mixta se diseñó una estrategia experimental consistente en la comparación de los resultados entre un grupo control (curso 20042005) y un grupo experimental (curso 2005-2006). Ambos grupos compartieron el profesorado. El grupo control recibió los contenidos del temario según la metodología clásica basada en la lección magistral y la realización de prácticas. En el grupo experimental se aplicó una metodología mixta de lección magistral, actividades realizadas en grupo y prácticas. El temario recibido por ambos grupos durante la lección magistral fue esencialmente el mismo, y sólo se vio reducido en el grupo experimental en aquellas horas destinadas a temas introductorios diversos y evitando el solapamiento de contenidos entre anatomía y fisiología. Estos temas fueron trabajados con lecturas previas en grupo por parte de los estudiantes del grupo experimental para potenciar el aprendizaje autónomo. Asimismo, con relación a las prácticas del grupo experimental, éstas fueron ampliadas 


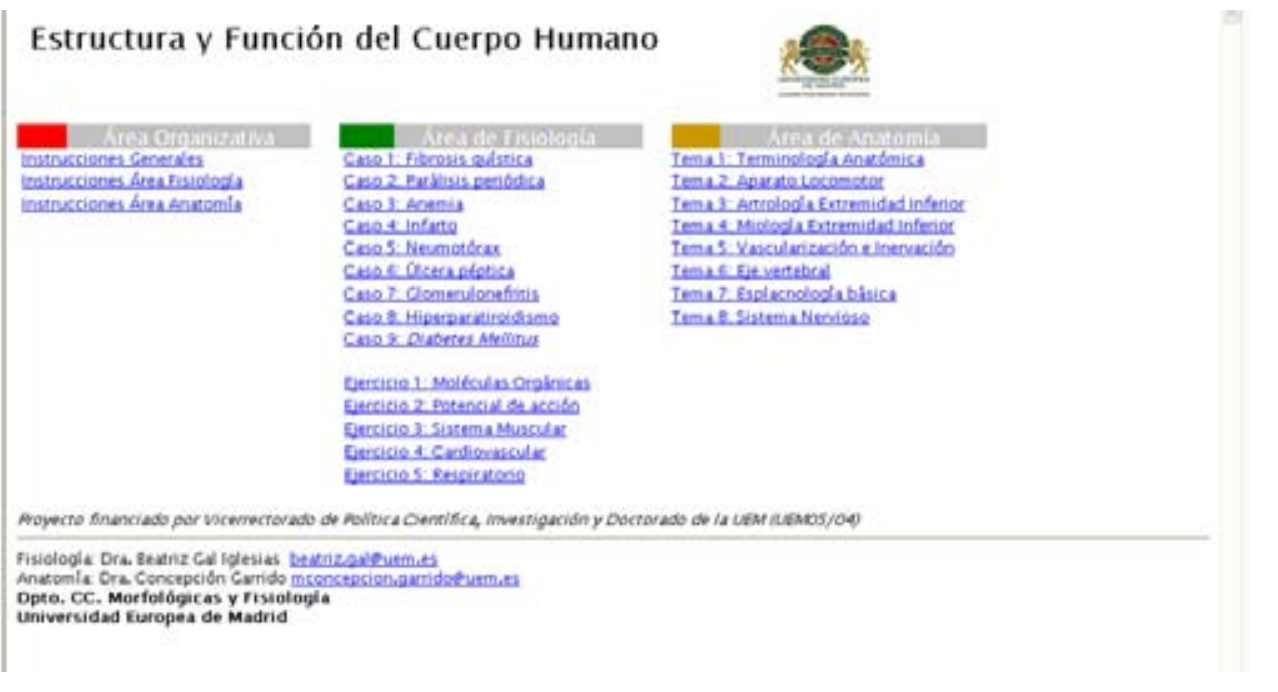

Figura 1. Página principal del CD organizado en tres áreas: organizativa, donde se explica el procedimiento; fisiología, con casos clínicos y ejercicios, y anatomía, con ejercicios estructurados en ocho temas.

con lecciones teórico-prácticas de diversos temas anatómicos y fisiológicos cuyo contenido teórico fue aligerado de la lección magistral.

La evaluación en el grupo control consistió en un examen tipo test, mientras que en el grupo experimental ésta se completó con la valoración del trabajo realizado en grupo y las competencias adquiridas. Las variables que se debían medir fueron la puntuación en los exámenes, la asistencia a clases y los resultados de la encuesta de satisfacción. Se midieron otras variables como edad, sexo, calificaciones escolares, etc. que permitieron concluir que los grupos eran homogéneos en todo, excepto en la metodología de enseñanza recibida.

La decisión de desarrollar el proyecto en la diplomatura de podología de la Facultad de Ciencias de la Salud (CSS) de la Universidad Europea de Madrid (UEM) se tomó basada en el bajo número de alumnos matriculados (10), que permitió aplicar la metodología mixta y trabajar de manera personalizada. Se constituyeron dos grupos de trabajo permanentes de cinco estudiantes. La planificación docente quedó establecida del siguiente modo: $50 \%$ horas presenciales, $20 \%$ horas de prácticas y $30 \%$ horas de trabajo en grupo (casos clínicos, problemas, ejercicios y revisión bibliográfica).

Al inicio del curso académico 2005-2006 (grupo experimental) se entregó a cada alumno la planificación docente, donde se incluyó la información relativa a los días de clase presencial, clase práctica o trabajo en grupo y se especificó la actividad docente concreta que se debía realizar. El objetivo de este procedimiento era facilitar la planificación del trabajo por parte del alumno para así fomentar el aprendizaje autónomo. Además, se elaboró un $\mathrm{CD}$ con todas las actividades docentes para realizar en grupo, tanto de la parte de fisiología como de la parte de anatomía (Fig. 1). El CD fue preparado íntegramente con FrontPage (Microsoft Office 2003), de manera que el formato es similar a Internet (lenguaje html) y la utilización y el acceso al material es sencillo e intuitivo (Inscripción en el Registro de la Propiedad Intelectual exp 12/ RTPI-001544/2006). Esta fase preparatoria es fundamental, pues está demostrada la importancia de la transparencia del diseño del curso y del sistema de evaluación para maximizar el rendimiento del estudiante [8]. Como se evidencia en el apartado de resultados, este aspecto del trabajo fue valorado muy positivamente en la encuesta de satisfacción (3,5/4; pregunta 3$)$.

Para el tratamiento estadístico de los datos se ha comprobado previamente el cumplimiento de las hipótesis exigidas por el modelo empleado, como el contraste de normalidad (KolmogorovSmirnov) o el de homogeneidad de varianzas (Levenne). En todos los casos aplicamos la técnica de contraste inferencial adecuada, paramétrica (ANOVA, MANOVA, $t$ de Student, etc.) o 
no paramétrica, menos exigente y concluyente, cuando no estaba indicada la utilización de una técnica de contraste de parámetros (Wilcoxon para dos muestras apareadas, Mann-Whitney para dos muestras independientes, Friedman para varias muestras relacionadas, $\chi^{2}$, etc.).

Un aspecto fundamental del trabajo fue el desarrollo de una encuesta de satisfacción sobre el uso de nuevas metodologías docentes (Tabla I). En la elaboración de la encuesta se tomaron en cuenta una serie de objetivos alrededor de los cuales se estructuraron un total de 12 preguntas cuantificables según una escala del tipo Likert de 1 a 4 ( 1 = nivel bajo; 4 = nivel alto) y dos preguntas abiertas.

\section{Resultados}

\section{Comparación 1: resultados del examen de tipo test en los cursos control y experimental}

En un primer análisis se compararon los resultados del examen de tipo test entre el grupo control y el grupo experimental. Para el grupo experimental se realizó un examen de tipo test similar al del curso control que puntuara el $60 \%$ de la nota. Esto permitía la comparación entre los dos grupos con arreglo a un sistema de evaluación común. En la tabla II se muestran los resultados del examen de tipo test en los dos cursos objeto de este estudio.

El análisis estadístico de los resultados del primer, segundo y tercer parcial de cada curso muestra que no hay diferencias estadísticamente significativas en las notas medias del examen tipo test en ambos cursos $(p>0,6)$. Sin embargo, en el curso control la nota media fue mejor para el segundo examen parcial, estabilizándose en el tercero. Por el contrario, esta tendencia se invierte en el grupo experimental, y las notas medias de segundo y tercer parcial son peores que las del primero (Tabla II). De hecho, la nota media del test en los tres parciales del curso experimental $(3,7 \pm 1,5)$ es significativamente peor que la del curso control $(5,1 \pm 2,3 ; t=7.757 ; p=0,007)$.

\section{Comparación 2: evaluación de nuevas metodologías (examen de competencias)}

El $40 \%$ restante de la nota de los parciales en el grupo experimental es el resultado de un nuevo modelo de examen asociado a las metodologías introducidas (casos, prácticas, trabajo en grupo y trabajo individual). Este diseño nos permitió evaluar comparativamente la conveniencia del nuevo baremo de medición del aprendizaje de la asignatura frente al tradicional examen de tipo test. En la tabla III se recogen las notas medias obtenidas por los alumnos del grupo experimental en el examen de competencias.

En un primer análisis se compararon los resultados del examen de nuevas metodologías en los tres parciales del curso experimental. Se observa una considerable evolución en las medias, aun cuando sus diferencias no son estadísticamente significativas $(p=0,345)$. La nota media aumenta en la segunda evaluación y se estabiliza en la tercera.

\section{Comparación 3: notas de los tres parciales en el curso control y el experimental}

La nota final de cada parcial en el caso del grupo control la constituye íntegramente la evaluación de tipo test. En el grupo experimental, la nota de cada parcial se calcula considerando los resultados del examen de tipo test y de las competencias según la proporción de 60 y 40\%, respectivamente. Puesto que cada método docente desarrolla habilidades y genera conocimientos que pueden representarse de modo diferente en los dos tipos de examen, decidimos estudiar el impacto de una evaluación mixta. En el siguiente gráfico (Fig. 2) aparecen comparados los resultados de las notas finales en los tres parciales en los grupos control y experimental.

Del análisis es notable señalar que a pesar de que la nota media, si se consideran únicamente los exámenes tipo test del grupo experimental, disminuyó a lo largo del mismo (Tabla II), la nota global del grupo experimental muestra una tendencia estable a lo largo de los parciales gracias a las competencias que los alumnos han adquirido, compensando así la disminución de la nota del test. Nótese, asimismo, que la variabilidad en las notas parciales del curso experimental son menores que en el grupo control. Esto muestra la importancia de mantener un método mixto de evaluación que compense las diferencias y estabilice el proceso de adquisición de conocimientos.

\section{Comparación 4: asistencia a clase}

Para evaluar el impacto de la actividad docente presencial y el trabajo en grupo en la aplicación de nuevas metodologías docentes se considera- 
Tabla I. Encuesta de satisfacción sobre el uso de nuevas metodologías para el aprendizaje de la asignatura 'Estructura y función del cuerpo humano' de la diplomatura de podología.

1. Dispone de tiempo suficiente para responder a las exigencias de trabajo

2. Tuvo acceso fácil a la información necesaria para cumplir con las tareas de trabajo

3. El material del curso ha estado bien preparado y se ha explicado cuidadosamente

4. La incorporación de esta metodología le aumentó su motivación por el estudio de la asignatura

5. Con esta nueva metodología logró aprender sobre los contenidos de la asignatura

6. La estructura de esta metodología está diseñada para estimular:
a) el trabajo en equipo
b) la capacidad de expresarse oralmente
c) la capacidad de expresarse por escrito
d) la capacidad de síntesis y comprensión de la información

7. Los conocimientos obtenidos con esta nueva metodología generan un mejor aprendizaje que el obtenido en una clase magistral

8. De los siguientes recursos utilizados, señale en qué grado ha contribuido para el mejor entendimiento del tema:
a) el uso del CD preparado para la asignatura
b) el estudio de casos clínicos
c) la búsqueda de bibliografía para la realización de los casos clínicos
d) la realización de ejercicios de fisiología
e) el uso de libros de texto
f) la realización de esquemas con la información relevante del tema
g) la discusión en grupo del tema
h) la cumplimentación de láminas anatómicas
i) el estudio de los modelos anatómicos
j) las exposiciones orales
k) la búsqueda de bibliografía

9. Los grupos de trabajo han sido una buena herramienta para estudiar, aprender la asignatura y compartir conocimientos e ideas

10. Todos los miembros del grupo han trabajado de manera equitativa

11. Los métodos de evaluación de este curso son equitativos y adecuados

12. Las clases de trabajo en grupo en horario lectivo han sido de utilidad y han estado bien organizadas

13. Nombre tres debilidades que a su juicio tiene esta metodología

14. Nombre tres fortalezas que a su juicio tiene esta metodología

Los ítems del 1 al 12 se valoran de 1 (menor nivel) a 4 (mayor nivel).

(Adaptada de Maldonado M, Vásquez M. Una experiencia de desarrollo de un proyecto de aprendizaje colaborativo como estrategia formativa: aprendo y capacito a través de casos clínicos. Universidad de Talca. Chile.)

Tabla II. Medias aritméticas y desviaciones estándares de los exámenes tipo test del curso 2004-2005 $(n=13)$ y 2005-2006 $(n=10)$.

\begin{tabular}{lcccccc}
\hline & \multicolumn{2}{c}{ Curso 2004-2005 (control) } & \multicolumn{2}{c}{ Curso 2005-2006 (experimental) } \\
\hline Evaluación & 1 . $^{\text {a }}$ & $2 .^{\text {a }}$ & $3 .^{\text {a }}$ & $1 .{ }^{\text {a }}$ & $2 .^{\text {a }}$ & $3 .{ }^{\text {a }}$ \\
\hline Media & 4,8 & 5,4 & 5,2 & 4,1 & 3,3 & 3,7 \\
\hline Desviación estándar & 2,3 & 2,3 & 2,5 & 1,3 & 1,3 & 1,5 \\
\hline
\end{tabular}




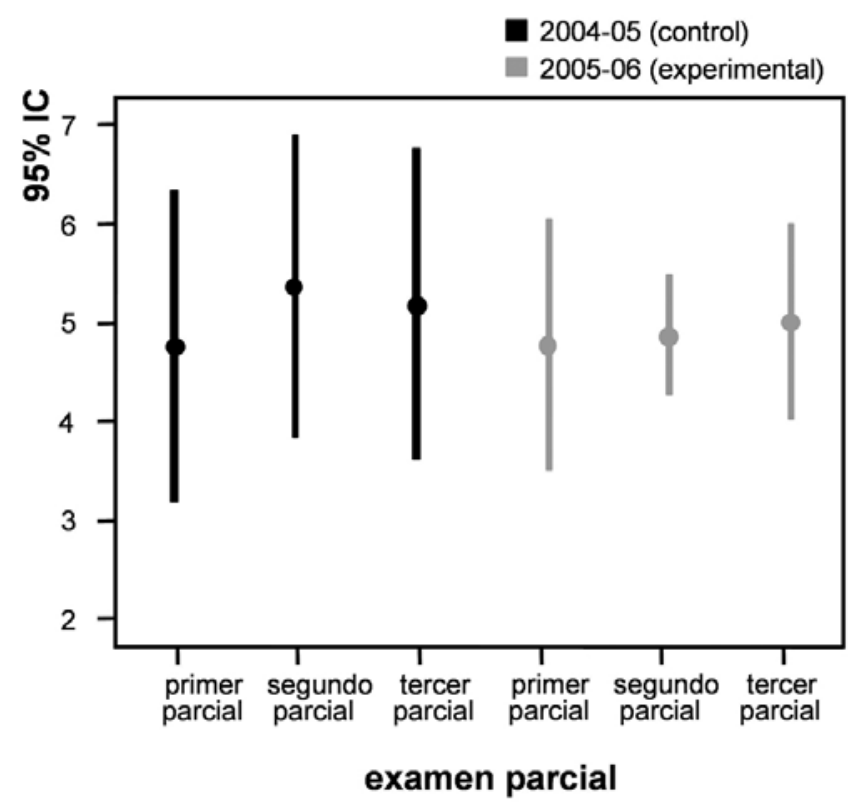

Figura 2. Intervalos de confianza al 95\% para las notas medias de los parciales en los grupos control y experimental.

ron los datos de asistencia, las clases magistrales, prácticas y las horas de trabajo en grupo. Para este propósito se estableció una escala desde 0 (nunca asiste) hasta 3 (siempre asiste).

El estudio muestra que la asistencia a clase se incrementó a lo largo del curso. La puntuación media fue de 1,8, 2,1 y 2,3 para los recuentos efectuados entre el principio del curso y el primer parcial, entre éste y el segundo y entre el segundo y el tercero, respectivamente.

\section{Comparación 5: regresión y correlación}

Para intentar confirmar las relaciones propuestas se han efectuado algunos análisis de regresión lineal y se ha medido el coeficiente de correlación lineal de Pearson.

En un primer estudio se compara la relación entre los exámenes de competencias y los de tipo test. No hay regresión lineal entre estas variables $(p=0,963$; coeficiente de correlación lineal $r=$ $-0,009)$ y se confirma que evalúan diferentes aspectos del proceso de aprendizaje. Sin embargo, los datos mostraron la existencia de una fuerte correlación entre la asistencia a las clases y la evaluación de competencias $(p<0,001$ y $r=$ 0,842; Fig. 3).
Tabla III. Medias aritméticas y desviaciones estándares (DE) de las evaluaciones de competencias efectuadas en el grupo experimental $(n=10)$.

\begin{tabular}{lccc}
\hline & $\begin{array}{c}\text { 2.a } \\
\text { evaluación }\end{array}$ & $\begin{array}{c}3 .^{\mathrm{a}} \\
\text { evaluación }\end{array}$ & evaluación \\
\hline Media & 5,8 & 7,2 & 6,8 \\
\hline $\mathrm{DE}$ & 3,0 & 1,8 & 1,2 \\
\hline
\end{tabular}

\section{Comparación 6: análisis de los resultados de las encuestas}

Como ya se ha mencionado, en la elaboración de la encuesta se plantearon 12 preguntas cuantificables según una escala del tipo Likert de 1 a 4 ( 1 = nivel bajo; $4=$ nivel alto $)$ y dos preguntas abiertas (Tabla I). Las preguntas cuantificables se organizaron en bloques destinados a valorar el grado de satisfacción con el nuevo material docente (preguntas 2 y 3 ), la percepción de un mejor aprendizaje (preguntas 4, 5 y 7), la valoración 


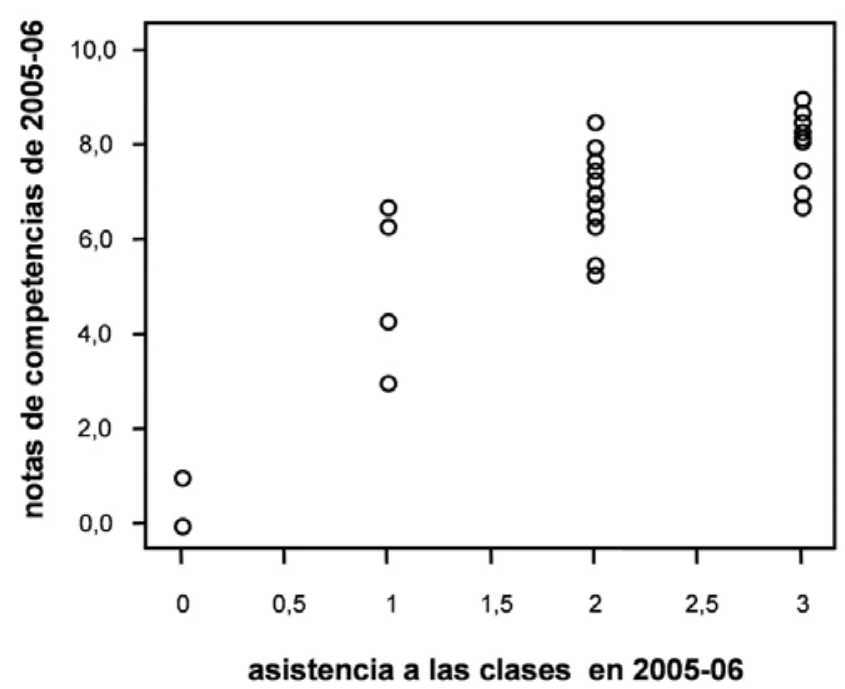

Figura 3. Diagrama de dispersión de la asistencia a clase frente a las notas obtenidas en las competencias.

relativa del peso de cada tipo de método de enseñanza en la formación de competencias (pregunta 6, 8 y 9), la gestión del tiempo (preguntas 1 y 12) y otros aspectos organizativos (pregunta 12) y evaluativos (pregunta 11). Las dos preguntas abiertas fueron concebidas para valorar las debilidades y fortalezas del método mixto según la percepción de los estudiantes.

En términos globales las medianas de todas las preguntas cuantificables estuvieron por encima de tres (sobre una escala máxima de 4). La mediana de las respuestas a las preguntas dirigidas a evaluar la percepción de los estudiantes de un mejor aprendizaje y desarrollo de competencias fue de 3,5. Este resultado estuvo en concordancia con las apreciaciones abiertas de los estudiantes sobre las ventajas del método mixto, donde las respuestas más frecuentes fueron:

- Fichas anatómicas y casos clínicos son muy buenas herramientas de aprendizaje.

- Desarrolla habilidades para hablar en público.

- Se aprende a trabajar en equipo.

En cuanto a las debilidades más importantes, los alumnos destacaron que el trabajo no se reparte equitativamente entre los miembros del grupo y que la dedicación que exige fuera de las horas de clases es elevada.

Entre las competencias más valoradas por los estudiantes destaca el trabajo en equipo y la ex- presión oral (media 3,5) sobre la capacidad de síntesis y comprensión de la materia (media 3 ). Los métodos más valorados para la comprensión de los temas y el desarrollo de competencias (pregunta 8) fueron la discusión en grupo de los temas y casos y la cumplimentación de láminas anatómicas (media 3,6). Entre los menos valorados destacan el uso de los libros de texto, la exposición oral y la búsqueda de bibliografía (media 2,8).

\section{Discusión}

En este trabajo se ha evaluado la utilidad de una metodología mixta en la enseñanza de la fisiología y anatomía tanto en los resultados del aprendizaje como en la adquisición de competencias de los alumnos, comparándolo con el método tradicional. La conclusión fundamental de este estudio es que con una aproximación mixta los alumnos adquieren habilidades y competencias a lo largo del curso, se estabiliza el aprendizaje a un nivel suficiente y se equilibran las diferencias individuales. Esto se manifiesta en una menor variabilidad de la nota media de los exámenes comparados con el grupo control.

Cuando se analizan detalladamente los resultados obtenidos, se aprecia que al comparar las 
evaluaciones tipo test entre el grupo control y el experimental la nota media en cada uno de los parciales es ligeramente peor en el grupo experimental. Esto podría sugerir que el método tradicional permite a los alumnos mejorar sus habilidades (cuantificadas por medio de la evaluación de tipo test) y que resulta más apropiado para la adquisición de conocimientos. Sin embargo, es importante valorar el impacto global del método tomando en cuenta no sólo este tipo de evaluación sino también el rendimiento con la nueva metodología. Al comparar los resultados del grupo experimental, sumando la nota obtenida al evaluarse el trabajo en grupo y el examen tipo test, se observó que la nota media mejoró del primer al segundo parcial y se estabilizó del segundo al tercero. Es interesante destacar que las variabilidades muestran mayor homogeneidad en el curso experimental, comparado con el control, que podría sugerir que las nuevas metodologías amortizan las puntuaciones más bajas y facilitan el aprendizaje de los alumnos con mayores dificultades. Además, dentro de la variabilidad, cabe destacar que ésta en la primera evaluación es mucho mayor que las de las otras dos evaluaciones, de forma que existen diferencias estadísticamente significativas entre las varianzas primera y tercera, lo que sugiere que los alumnos del grupo experimental aumentaron su nivel competencial a lo largo del curso, equilibrando las diferencias como parte del proceso de aprendizaje.

Por otro lado, con el análisis de regresión y correlación se puede apreciar que no hay regresión lineal entre los exámenes tipo test y los que evalúan la metodología mixta. Esto confirma que ambos tipos de examen evalúan diferentes aspectos del aprendizaje y sugiere la importancia de mantener un método mixto de evaluación que compense las diferencias. Sin embargo, sí se detecta una fuerte regresión lineal entre la asistencia a clase y la evaluación de las competencias, que confirma la extraordinaria importancia de este factor para incrementar el nivel de competencias a través de la supervisión del proceso de aprendizaje por parte del profesor.

Es importante considerar el hecho de que la limitación del número de datos, lo que por otra parte era inevitable y conveniente para el desarrollo de este estudio, condiciona la obtención de diferencias estadísticamente significativas. Este hecho obliga a considerar los resultados cuan- titativos como posibles tendencias cuya confirmación estadística requerirá la utilización de tamaños de muestras adecuados con arreglo a la teoría general de muestras. Asimismo, la imposibilidad de elección aleatoria de las muestras en el presente análisis aconseja la prudente interpretación de las técnicas inferenciales utilizadas.

Por último, conviene destacar la alta satisfacción de los alumnos con la metodología mixta, puesta de manifiesto con los resultados de la encuesta realizada al finalizar el curso, que evidencia una alta percepción del aprendizaje por parte de los alumnos. Esto configura un importante factor subjetivo que potencia la actitud de los estudiantes ante el proceso formativo. Sin embargo, nos gustaría destacar la necesidad de profundizar en el diseño de métodos que aseguren un incremento en la comprensión de la materia a través del obligado uso de los libros de texto y la búsqueda bibliográfica, dos de las herramientas más importantes para desarrollar la aptitud formativa continuada de los profesionales de ciencias de la salud.

\section{Bibliografía}

1. Lujan HL, Di Carlo SE. Too much learning, not enough learning: what is the solution? Adv Physiol Educ 2006; 30: 17-22.

2. Bolonia, 1999. URL: http://www.aneca.es/modal_eval/ docs/declaracion_bolonia.pdf.

3. González J, Wagenaar R. Tuning educational structures in Europe. Pamplona: Universidad de Deusto; 2003.

4. Gage N, Berliner D. Educational psychology. Boston: Houghton Mifflin; 1998.

5. Hudson JN, Buckley P. An evaluation of case-based teaching: evidence for continuing benefit and a realization of aims. Adv Physiol Educ 2004; 28: 15-22.

6. Walters MR. Case-stimulated learning within endocrine physiology lectures: an approach applicable to other disciplines. Adv Physiol Educ 1999; 276: 74-8.

7. Waters JR, Van Meter P, Perrotti W, Drogo S, Cyr RJ. Cat dissection vs. sculpting human structures in clay: an analysis of two approaches to undergraduate human anatomy laboratory education. Adv Physiol Educ 2005; 29: 27-34.

8. Hendry GD, Lyon PM, Gordon J. Students evaluation of expert and non expert problem-based learning tutors. Med Teach 2002; 24: 544-9. 